\title{
Implementation of Stellarator of Costa Rica 1 SCR-1
}

\author{
V.I. Vargas, J. Mora, C. Otárola, E. Zamora, J. Asenjo, A. Mora, and E. Villalobos \\ Plasma Laboratory for Fusion Energy and Applications \\ Instituto Tecnológico de Costa Rica \\ Cartago, Costa Rica \\ Corresponding author e-mail: ivanvargasblanco@gmail.com.
}

\begin{abstract}
The SCR-1 is a small modular stellarator designed, constructed and is being implemented at Instituto Tecnológico de Costa Rica. The project is its final phase of commissioning and it is expected to be operational in June 2015. This article aims at summarizing the SCR-1 project from the engineering and manufacturing point of view, particularly highlighting the construction of the toroidal vacuum vessel and the modular coils.
\end{abstract}

Keywords-Magnetic confinement; Stellarator; low shear configuration; small modular stellarator

\section{INTRODUCTION}

It has been recently demonstrated, contrary to what it was expected, that for tokamaks operating at fixed portions of density and beta, fusion gains depend mainly on the absolute level of fusion power and energy confinement and just lightly on the device size [1]. Also, the concept of a stellarator, in contrast with a tokamak, allows operating continuously without large plasma current that is the potential danger of so-called disruptions. From this, we can conclude, first, that the size of the device is not as relevant as expected; and, second, the concept of a stellarator presents increasingly more advantages than a tokamak.

Since 2009, the Instituto Tecnológico de Costa Rica started a research project on stellerators which aims at designing, constructing and implementing the first device of this technology in Latin America. Small stellarators present clear advantages related to total cost of the project which allows with a relatively low investment train human resources and contribute to the physics and engineering of such devices within the fusion scientific community.

The SCR-1 is a small modular Stellarator for magnetic confinement of plasma developed by the Plasma Laboratory for Fusion Energy and Applications of the Instituto Tecnológico de Costa Rica (ITCR). SCR-1 will be a 2-field period, small modular stellarator with an aspect ratio of $>$ 4.4; a low shear configuration with core and edge rotational transform equal to 0.32 and 0.28 . It will hold plasma in a 6061-T6 aluminum, torus-shaped, vacuum vessel with an minor plasma radius of $54.11 \mathrm{~mm}$; a volume of 13.76 liters $\left(0.01 \mathrm{~m}^{3}\right)$; and, major radius of $\mathrm{R}=238 \mathrm{~mm}$. Plasma will be confined in the volume by an axis magnetic field of $43.8 \mathrm{mT}$ generated by 12 modular coils with 6 turns each, carrying a current of 767.8 A per turn providing a total toroidal field (TF) current of $4.6 \mathrm{kA}$-turn per coil. The coils will be supplied by a bank of cell batteries of $120 \mathrm{~V}$. Typical length of the plasma pulse will be between $4 \mathrm{~s}$ to $10 \mathrm{~s}$. The SCR-1 plasmas will be heated by $\mathrm{ECH}$ second harmonic at 2.45 $\mathrm{GHz}$ with a plasma density cut-off value of $7.45 \times 10^{16} \mathrm{~m}^{-3}$. Two magnetrons with a maximum output power of $2 \mathrm{~kW}$ and $3 \mathrm{~kW}$ will be used.

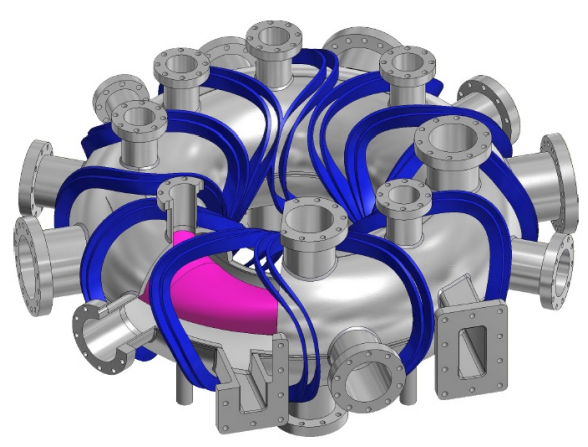

Fig. 1. SCR-1 vacuum vessel drawing.

This article is divided in engineering aspects, design characteristics, vacuum vessel, modular coils, and peripherical systems and support. Finally we will mention the current status of commissioning and the different tests already done and the ones expected to be completed in the stellarator.

\section{ENGINEERING ASPECTS}

The stellarator vacuum vessel along with its coil support, coils and ports were completely constructed in Costa Rica by the Instituto Costarricense de Electricidad (ICE), after hiring their services. All ancillary components and peripherical systems were chosen according to design criterion and they were bought new. Additional stellarator support structures for the peripherical systems (ECH system, Gas Injection System) and the diagnostics were constructed in Costa Rica using 304LN austenitic steel with relative magnetic permeability below 1.01 .

The engineering phase, previous to the construction of SCR-1, was divided in the following steps [2]:

-Predesign

-Design

-Design review

-Detailed design within the manufacturing contracts 
All important data in which the SCR-1 engineering is based, for example, the required dimensions, coils parameters, conductors electric resistance, magnetic fields, inductances, forces, power source requirements, thermal resistance of coil cabling and short-circuit current, were revised twice independently. All calculations that used finite elements with COMSOL Multiphysics were also checked twice. The contractor that manufactured the stellarator (vacuum vessel, coil support, coils and ports) participated since the "design review" phase. At that moment, it was defined the first agreement with general requirements which included the hiring of services to research the final method of construction. At the beginning of "detailed design" phase, it was necessary to define a new agreement with more detailed construction specifications. The reduced size of the SCR-1 presented a great challenge during the construction. It was necessary to construct three life-size, toroidal, aluminum vacuum vessels: some of them with one or two ports for testing to be able to determine the construction method that ultimately was going to be used. Testing included coil construction tests, and welding tests on the ports and on the vacuum vessel.

\section{DESIGN CHARACTERISTICS}

SCR-1 main design characteristics are the following:

- Small size (major radius $\mathrm{R}=238 \mathrm{~mm}$ ).

- Modular stellarator (modular coils).

- 2-field period

- Low shear configuration

- Heating frequency of $2.45 \mathrm{GHz}$

- Individual support of main components

- No magnetic field coils inside the vacuum vessel

- Simple geometry of vacuum vessel

- No water-cooled copper coils

- Relative magnetic permeability below 1.01

\section{VACUUM VESSEL}

SCR-1 vacuum vessel was manufactured with two 6061T6 aluminum cylindrical building blocks. Each block had the following dimensions: $110.00 \mathrm{~mm}$ height and $694.80 \mathrm{~mm}$ diameter. Although using austenitic 304L grade stainless steel was analyzed, it was discarded because of the difficulty to manufacture parts according to the device dimensions and because it increased greatly project costs. After extracting for each aluminum block a central cylinder of $257.20 \mathrm{~mm}$, it was used a three-axis CNC machine with ball mill to create a channel of toroidal geometry with a radius of $94.42 \mathrm{~mm}$ in each block, as shown in Figure 1a. Internal surface was polished and it was obtained $a R_{a}=0.3$. Two holes were previously perforated in each block where there are ports for gases to escape during welding processes and a coupling channel was mechanized which allowed joining both pieces and weld with TIG technique, both in the internal string and in the external coupling (See Figure 1b). Once this was done, thanks to the CNC milling machine with a ball mill the external part of the vacuum vessel was mechanized which left a finish as shown in Figure 1c. It was finally obtained a vacuum vessel with $10 \mathrm{~mm}$ thickness and a volume of $0.0418 \mathrm{~m}^{3}$, an external radius of $364.1 \mathrm{~mm}$, an internal radius of the central circle of $112.1 \mathrm{~mm}$, a major radius of $R=238.1$ $\mathrm{mm}$, and, as mentioned previously, the internal radius of the vacuum vessel is $94.42 \mathrm{~mm}$. When this was finished, a T6 thermal treatment was applied again to the vacuum vessel to guarantee rigidity and penetrating fluids tests were performed.
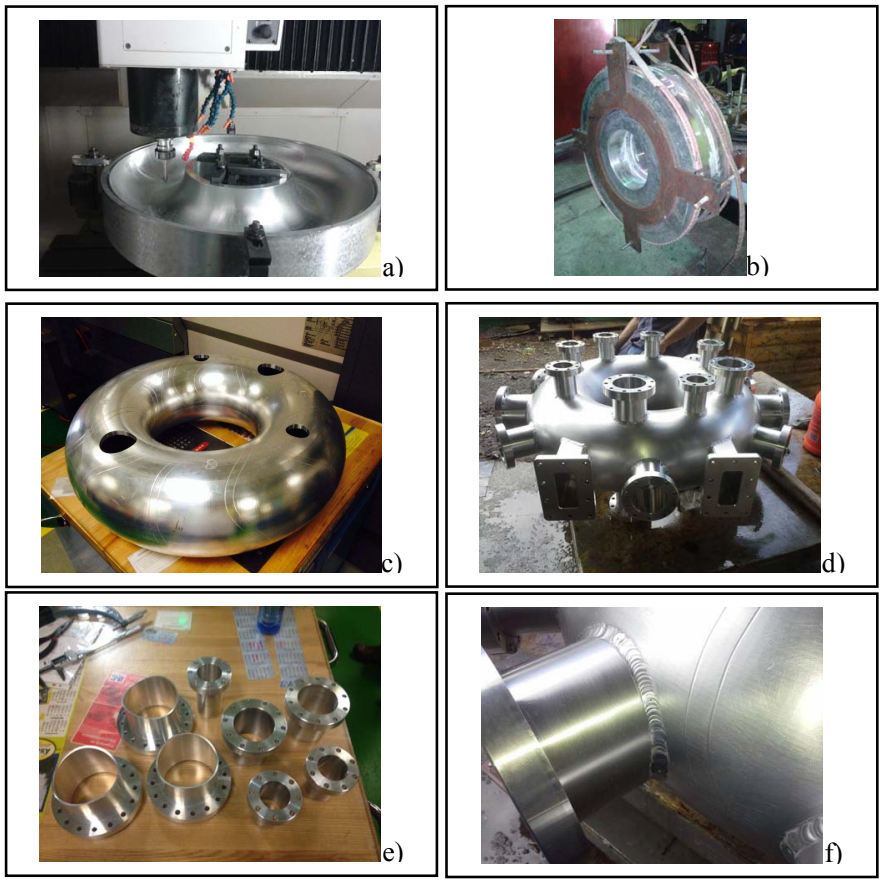

Fig. 2. Stages of the vacuum vessel construction.

The perforations for CF ports in the superior, inferior and middle part of the vacuum vessel were done using a CNC machine. Each port was mechanized according to design dimensions. The vacuum vessel has 24 conflat ports: three of the ports are CF of 6", 10 are CF of 4-5/8", and 9 are CF of $3-3 / 8$ ". Two rectangular ports, internal dimensions of $90 \mathrm{~mm}$ x $40 \mathrm{~mm}$, were added to accommodate future needs (See Figure 1.d). It was decided to use, for all ports, the same CF aluminum design of the vacuum vessel with Cusp Confinement Plasma called MPDX (Madison Plasma Dynamo Experiment) from the University of Wisconsin, Madison [3] (See Figure 1e). Nowadays, Viton seals (O-ring seals) and vacuum grease are used in the ports. The port covers, viewports and other components coupling to the CF ports were bought in stainless steel according to standard design and dimensions of vacuum component suppliers.

The welding process was certified by manufacturer to AWS welder and it was used a standard procedure as AWS A5.3 and AWS A5.10, which specify aluminum welding procedure (See Figure 1f). 


\section{MODULAR FIELD COILS}

\section{A. Coil support system}

In the predesign, design, and design review phases, different ideas on how to place the coils above the vacuum vessel, methods as the one indicated on [4], were considered. Finally in the detailed design phase, it was decided to use coil support or guides that were welded to the vacuum vessel and with the specific shape of each; which should leave a channel in which a conductor wire would be placed. The fabrication of coil supports and guides was done through a cast procedure. This method was chosen because it allows building pieces with the exact shape. Now, to create the casting molds a 3D printer, Dimension DST1200ES, was used. It has a print volume of $254 \times 254 \times 305 \mathrm{~mm}$ and a print precision of $0.254 \mathrm{~mm}$, which is more than acceptable for the creation of the supports because the defined tolerance in the design phase for this process was $+/-2 \mathrm{~mm}$.

The SCR-1 magnetic configuration has three coils that are repeated four times; in other words, it was only needed to manufacture three supports in the 3D printer and manufacture three casts based on this model. Figure $2 \mathrm{a}$ shows three supports selected to be printed. Since the dimensions of the support exceeded the maximum the printer could print ( $254 \times 254 \times 305 \mathrm{~mm})$, it was needed to divide each coil support wall or guide in 4 parts, as shown in Figure $2 b$. This decision influences the welding of the supports to the vacuum vessel because since they were divided in 4 parts it is easier to handle the temperature of each piece and avoid the thermal contractions during the welding process.

Once supports were ready with the $3 \mathrm{D}$ printer, the casting mold was created and the cast started. Casting parameters and necessary calculations were determined by manufacturer. Figure 2c shows the result of the 3D print pieces and Figure $2 \mathrm{~d}$ shows aluminum casting pieces that were welded to the vacuum vessel.

The following step was to place the supports in the vacuum vessel, but first it was necessary to mark accurately where the supports were to be welded. It was decided to use a CNC milling machine with a ball mill (to allow to always making contact with the tangency point) to create a channel of $0.2 \mathrm{~mm}$ with the coil shape reflected on the vacuum vessel for the welder to know where to place the piece and weld it. Figure 2e shows the marking on the vacuum vessel.

Due to manufacturer limitations related to only having CNC milling machines of three and four axis, work was completed in two parts, in other words, half coils were marked and, then, the toroid was turned to mark the other half, using a three axis CNC. CNC machine precision is 0.1 $\mathrm{mm}$, which didn't increase the possibility of losing precision on final position of coil supports or guides. The final result is shown in Figure 2f.

Verification testing on mark positioning were performed where coil support were placed. It was used a vacuum vessel 3D model and a CMM machine to measure the coordinates. This tool allows reviewing points of interest with a probe and it shows coordinates in 3D. This coordinates then are compared to the 3D model to determine errors.

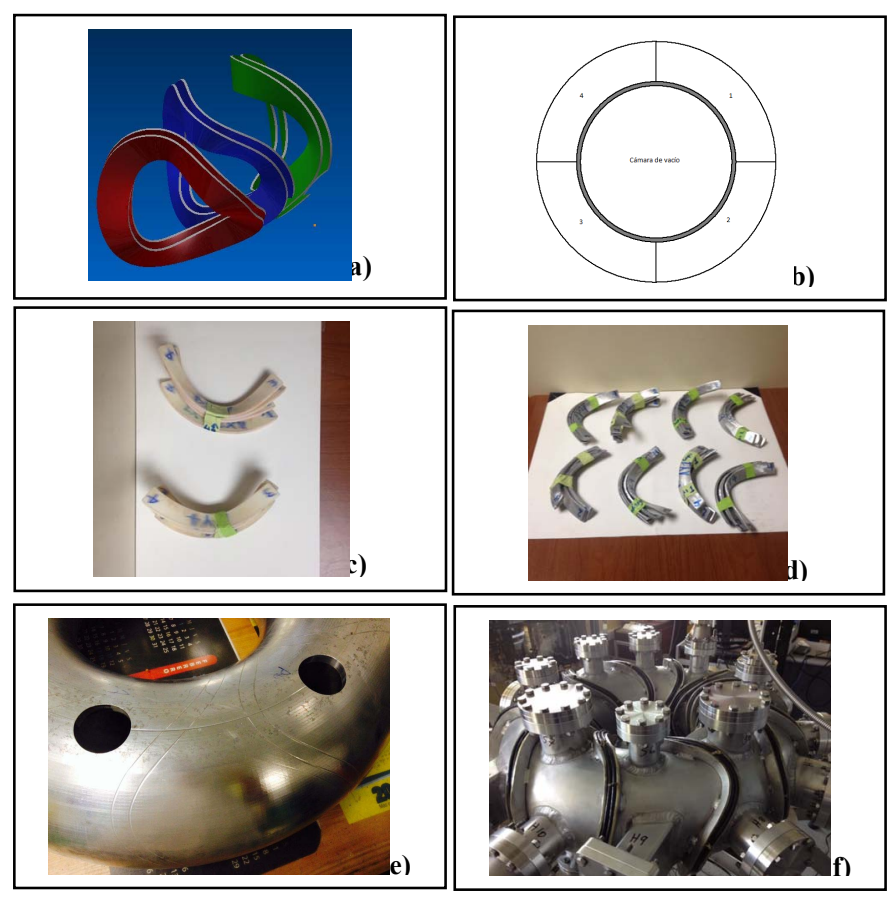

Fig. 3. Stages of the coil support construction.

\section{B. Magnetic coil system}

The SCR-1 magnetic field of confinement will be generated by 12 copper modular coils, whose supports and positioning were done according to the beforehand explanation. Coil geometry was obtained by engineer Vicente Queral [4], from CIEMAT, in Spain. Each modular coil will have 6 turns, made of AWG\#4 wire, and a current of 767.8 A per turn providing a total toroidal field (TF) current of $4.6 \mathrm{kA}$-turn per coil.

Thermal transfer simulations were performed in modular coils [5]. The thermal behavior of copper wire by electrical current passing in the modular coil was simulated using COMSOL Multiphysics software and other methods. Temperature, resistance, voltage and power calculations as a function of time were performed for the electrical circuit under different wire configurations per modular coil to select the power supply taking into account the available budget. The wire configurations of 1,2 and 4 turns per coil were discarded due to the high price of the power supply (USD > $50 \mathrm{k} \$$ ). Also the wire configurations of 8 and 12 turns were also discarded because the clashes occurring between close coils when the height per coil increased. Finally it was decided that 6 turns per coil was best. In this case, temperature changes from $20^{\circ} \mathrm{C}$ to $70{ }^{\circ} \mathrm{C}$ in $8 \mathrm{~s}$, and thermal capacity of heat shrink tubing that wraps the conductor cable is $125{ }^{\circ} \mathrm{C}$. Three thermo-coupling were placed in different coils in expected points of highest temperature due to closeness with coils. Active cooling systems were considered as a possible solution for the temperature rise of the coils but discarded due to the constraints imposed by the available 
space and complex geometry of the coils, though remain a possibility for future upgrades.

Coiling process was performed as in [4], guaranteeing coil input and output will be one next to the other and to the same side. To SCR-1, current inputs and outputs were left towards

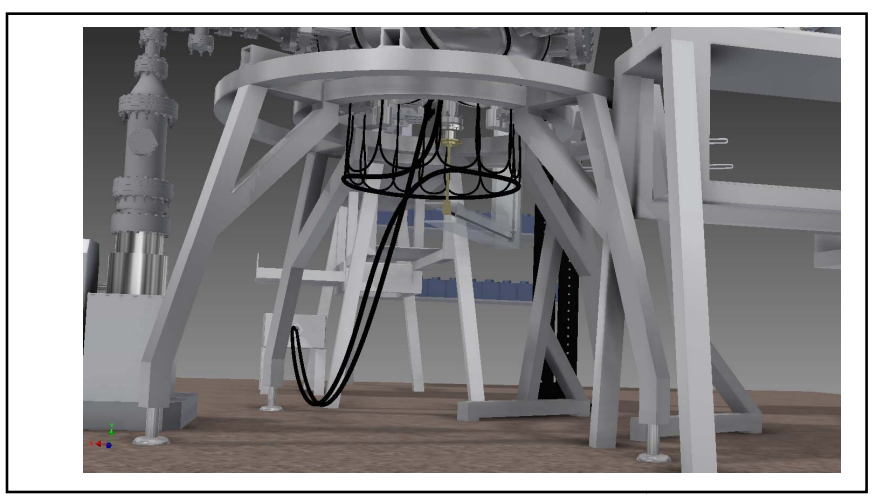

Fig. 4. Peripherical wires to the coils.

the bottom with the objective of avoiding limiting the space to place equipment and diagnostics in the upper part of the stellarator (See Figure 4).

\section{Coils connection and electric current regulator}

Modular coils were connected in series with heterogeneous welding, using tin to create joints in cabling ends. This allows reducing total resistance of 12 coils to 60 $\mathrm{m} \Omega$ by not using connectors among the coils. Once the coils were connected, they were welded to the end of an AWG \#4 cable, AWG \#0 copper cable, and it was returned through where the coils are connected in series to cancel magnetic fields generated by peripherical wires to the coils. Cabling inputs and outputs of each coil are next to each other and separated by $30 \mathrm{~cm}$ from the vacuum vessel also to cancel magnetic fields generated by conductors (See Figure 4).

To keep a constant current during a period of time less than $10 \mathrm{~s}$, in spite of ohmic heating in coil conductor cable, it was designed an electrical current controller. This features a low cost, efficient power circuit and a PID digital control with a speed algorithm which reduces harmonics to avoid creating errors in the magnetic field of the Stellarator. For the design of the current controller, it was used the concept of a Buck converter and it includes 9 IGBTs, 9 R2 resistances with a value of $0,1 \mathrm{Ohm}, 9 \mathrm{R} 1$ resistances with a value of 0,3 Ohm, 9 capacitors of 0,1 microfarads, 9 diodes and 9 L coils of 315 microhenries as shown in Figure 5. For this circuit, 9 Buck converters were used in parallel, to reduce the power that each component had to handle, which in turn cut down construction costs. Besides lowering costs, there is a technical reason to use more than one IGBT: diminish the magnitude of harmonics. In this case, three frequencies were used that are not multiples among themselves. $27 \mathrm{kHz}, 23$ $\mathrm{kHz}$ and $19 \mathrm{kHz}$ were selected. Clearly, two of them are prime numbers which guarantees that they are not multiples among themselves, and the third frequency is chosen with a difference of $4 \mathrm{kHz}$ regarding the last, to avoid that, in the spectrum, they become so close that they can combine. The need to reduce harmonics in output current is due to the effect that these can have on plasmas; as a result it is better to have more output frequencies than an intense magnitude. In the controller, it was included a PID control with a speed algorithm to diminish harmonics. To verify its operation, it was used a PSIM software, which

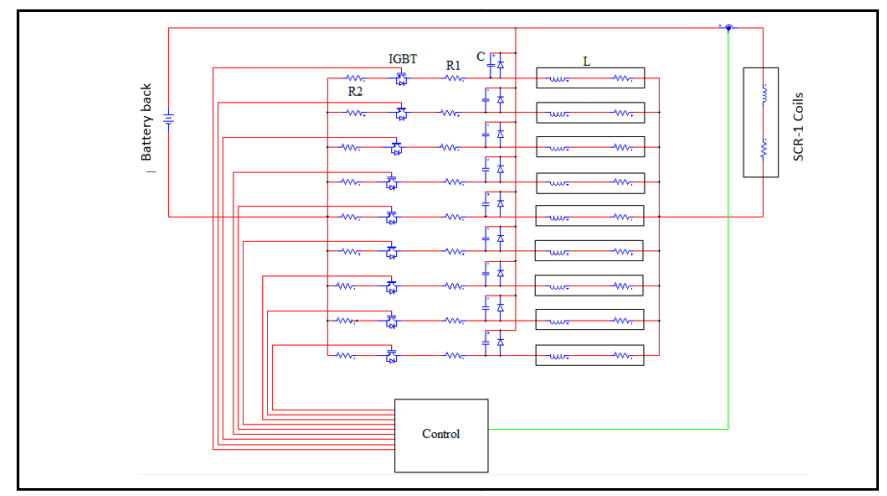

Fig. 5. Electric circuit of electrical current controller.

allows to integrate the power circuit and the system control in blocks and $\mathrm{C}++$ language.

\section{PERIPHERICAL SYSTEMS AND SUPPORT STRUCTURE}

\section{A. ECRH system}

The system has two magnetrons of $2 \mathrm{~kW}$ and $3 \mathrm{~kW}$ with a frequency of $2.45 \mathrm{GHz}$. Each one has a power supply, magnetron head, isolator, directional coupler, and tuner. One WR340 rectangular-to-circular wave guide transition was especially designed to couple with a CF port of 6 inches. Two CF ports of 6 inches have quartz viewports with optimum thickness for microwave transmission. More information about the ECRH system of SCR-1 stellarator can be seen in [5].

\section{B. Gas injection system}

Gas injection to the devise will be achieved with a mass flow controller (MFC-ALTA) with maximum scale of 20 SCCM, and a resolution of 2 SCCM and a response time of 1s. Two gas injection lines are included, with filters and connections to the vacuum system, besides a line exclusively to vent the system with $\mathrm{N}_{2}$.

\section{Vacuum pumping system}

The main vacuum system component is the vacuum pumps group, which is composed by one scroll mechanical pump able to reach $10^{-4}$ Torr, and one turbo-molecular pump that can then achieve a further reduction in pressure to $10^{-10}$ Torr. An automated pump system was chosen, integrating the controllers of both pumps. This group has additional equipment such as RS485 communication, vacuum convectron, ion gauge sensors and RGA (Residual Gas Analyzers) for possible gas leak issues. 


\section{Power supply}

The electrical supply system will consist of an array of 60 lead-acid electrochemical cells (battery bank); each cell will have a nominal voltage of $2 \mathrm{~V}$ and an electrical storage capacity of $150 \mathrm{~A}-\mathrm{h}$. The battery bank will have a voltage of $120 \mathrm{~V}$ and will be able to deliver a current of at least the required 767.8 A. As mentioned before, between the power source and the stellarator, there would be a current controller,

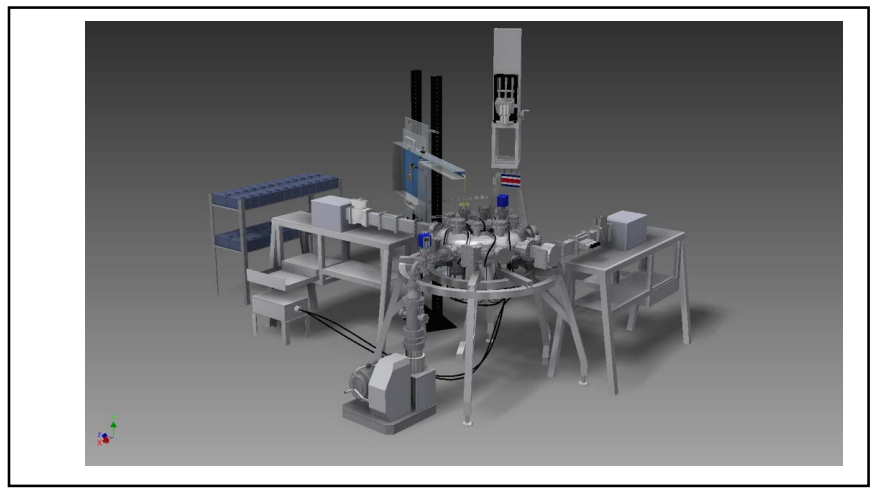

Fig. 6. Drawing of all peripherical systems and support structures.

and the limit on the duration of SCR-1 discharges will be determined in first place by maximum temperature of 125 ${ }^{\circ} \mathrm{C}$, which can be resisted by the thermo shrinkable tubing wrapping conductor cabling.

\section{E. Control system}

Device data acquisition and control will be performed with a PXI platform, a NI PXIe-1082, and high speed data acquisition modules (1.25 to $2 \mathrm{MS} / \mathrm{s}, 16 \mathrm{bits})$, industrial digital E/S modules and PCI high performance serial interfaces.

\section{F. Support structure}

All peripherical system support structures (ECRH system, diagnostics) and the support base of SCR-1 vessel were constructed in Costa Rica with 304LN austenitic steel with relative magnetic permeability below 1.01. Two rectangular tables were designed and built for the ECRH system, a circular table with four open support points for the SCR-1, a special support for the Langmuir probe and a Plexiglas support for the microware heterodyne interferometer as shown in Figure 6 were also created. In addition, a support table for the optical spectrometer was designed.

\section{STATUS OF ASSEMBLY ON SITE, TEST PROGRAM AND COMMISSIONING TESTS}

To this date the covers, viewports, vacuum sensor, vacuum pumps, etc, have been placed. The first vacuum testing suggests pressures of $10^{-7}$ Torr in the toroidal vessel. ECRH systems of $2 \mathrm{~kW}$ and $3 \mathrm{~kW}$ have been tested and operated thanks to the control system indicated above. There have been hydrogen gas injection testing and an injection pattern has been determined for the first discharge. The battery bank is operating and charging; it is ready to be used in the first tests to provide power supply to the coils. Electrical current controller is being assembled and it is expected that by June 2015, it will be operational to be able to do the first plasma discharge. Once all peripherical systems finish the start up, they will undergo magnetic field mapping tests, following the procedure stated in [6]. For the second half of 2015, it is expected to have the first electron density and temperature measures using Langmuir probe and optical spectrometer.

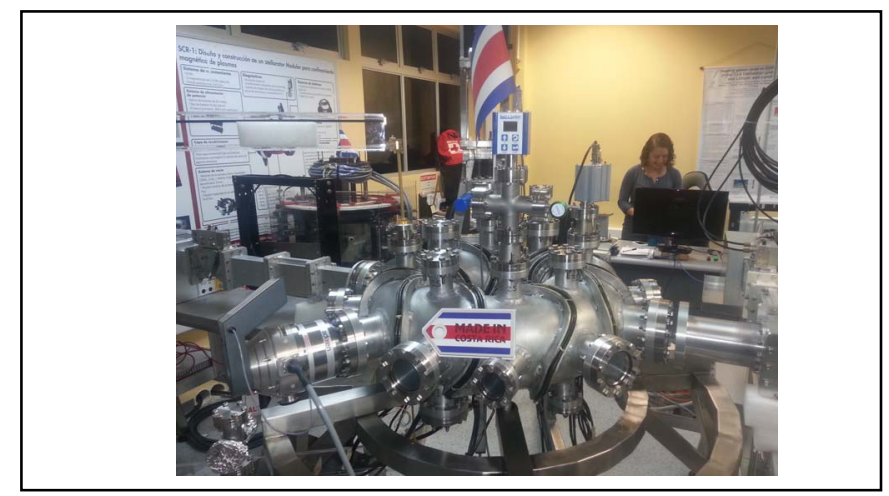

Fig. 7. Status of assembly on site of Stellarator SCR-1.

\section{CONCLUSIONS}

A small modular stellarator has been designed and constructed and it is under the implementation phase at Instituto Tecnológico de Costa Rica (See Figure 7). To this date the aluminum vacuum vessel has surpassed the values of expected low pressures. It was implemented an innovative method to construct modular coils using 3D printer and casting Al. The peripherical systems as ECRH, gas injection, vacuum pumping system, power supply, control system and support structure are ready and operational. It is expected that in June 2015 the start up of the electrical current controller is finished and with that being able to do the first plasma discharge, besides being able to do magnetic field mapping testing.

\section{ACKNOWLEDGMENT}

The authors would like to thank the engineer Vicente Queral and the Laboratorio Nacional de Fusión, CIEMAT, in Madrid, Spain, for the support provided throughout this project. We also deeply thank the Instituto Tecnológico de Costa Rica, which through the Vicerrectoría de Investigación funded this project and all the engineers from Instituto Costarricense de Electricidad (ICE) who participated in the toroidal vacuum vessel and modular coils construction.

\section{REFERENCES}

[1] a E. Costley, J. Hugill, and P. F. Buxton, "On the power and size of tokamak fusion," Nucl. Fusion, vol. 033001, p. 33001. 
[2] M. Blaumoser, "Engineering, fabrication, tests and status of the Spanish Stellarator TJ-II," Proc. 16th Int. Symp. Fusion Eng., vol. 2, pp. 1062-1065, 1995.

[3] M. M. Clark, A. Eckhart, K. Flanagan, J. Milhone, A. Rasmus, I. Reese, J. P. Wallace, D. Weisberg, and C. B. Forest, "Fabrication of a Vacuum Vessel for a Cusp Con nement Plasma using Cast Al," in 54th Annual Meeting of the APS Division of Plasma Physics, October 29-November 2, 2012, p. 1.

[4] V. Queral, "Coil fabrication of the UST_1 modular stellarator and potential enhancements," Fusion

Eng. Des., vol. 88, no. 6-8, pp. 683-686, Oct. 2013.

[5] V. I. Vargas, J. Mora, J. Asenjo, E. Zamora, C. Otárola, L. Barillas, J. Carvajal-Godínez, J. González-Gómez, C. Soto-Soto, and C. Piedras, "Constructing a small modular stellarator in Latin America," J. Phys. Conf. Ser., vol. 591, p. 012016, 2015.

[6] P. Drewelow, T. Bräuer, M. Otte, F. Wagner, and A. Werner, "Three-dimensional photogrammetric measurement of magnetic field lines in the WEGA stellarator," Rev. Sci. Instrum., vol. 80, no. 12, pp. 1-8, 2009. 